\title{
EVALUACIÓN Y REGULACIÓN DE PARÁMETROS EN UN EQUIPO DISEÑADO PARA LA DISMINUCIÓN DE CROMO(VI), PROVENIENTES DE LA INDUSTRIA DE RECUBRIMIENTOS GALVÁNICOS
}

\section{EVALUATION AND PARAMETERS REGULATION IN EQUIPMENT DESIGNED FOR DECREASE OF CHROMIUM(VI), COMING FROM GALVANIC COATINGS INDUSTRY}

\author{
Gema Eunice Acosta Niño \\ M.Sc. Química, Profesor Asociado, Facultad de Ciencias Básicas y Aplicadas, \\ Universidad Militar Nueva Granada, Bogotá D.C., Colombia, Investigador grupo TAYQUITE?. \\ gema.acosta@unimilitar.edu.co
}

Alejandro Bourdón García

Químico, Investigador externo, Profesor catedrático Universidad Militar Nueva Granada, Bogotá D.C., Colombia. jose.bourdon@unimilitar.edu.co

Carlos Andrés Coy Barrera

PhD. Química, Profesor Asociado, Facultad de Ciencias Básicas y Aplicadas, Universidad Militar Nueva Granada, Bogotá D.C., Colombia.

carlos.coy@unimilitar.edu.co

Fecha de recepción: 12 de enero de 2013

Fecha de aprobación: 22 de mayo de 2013

\section{RESUMEN}

Con el fin de generar los medios necesarios para poder llevar a cabo las pruebas de remoción de cromo(VI) en aguas residuales de la industria galvánica, se realizó la captura de datos a partir de prototipo de equipo inicial a escala de laboratorio para conseguir el objetivo principal, disminuir la cantidad de metales en aguas residuales contaminadas con diferentes metales.

Es así que con los datos obtenidos en el presente estudio se plantearon algunas modificaciones necesarias para mejorar las condiciones y eficiencia del equipo y aumentar las posibilidades de uso mediante la determinación de los parámetros de trabajo usando la técnica de electrocoagulación.

Los parámetros analizados fueron: voltaje, intensidad de corriente, conductividad, $\mathrm{pH}$ y temperatura durante una hora de funcionamiento del reactor.

A lo largo del texto se pueden observar los cambios realizados sobre el prototipo inicial, cuyo concepto principal es el manejo

Tecnologías Ambientales y Química Teórica, grupo de investigación adscrito a la Facultad de Ingeniería de la Universidad Militar Nueva Granada. 
de la electrocoagulación, para disminuir la cantidad de metales en aguas residuales. Un resultado final de interés a analizar entre otros, es el consumo de energía, pues basándose en este consumo se calculó el valor final del gasto energético versus el costo ambiental, lo que sin duda resulta ser un aporte importante para su uso en la remoción de metales pesados y abre la posibilidad de continuar con estudios posteriores de este tipo para la elaboración de nuevos reactores mucho más eficientes y útiles en la industria en general, y como respuesta, ayudará bastante en la disminución de la contaminación ambiental.

Palabras clave: Electrocoagulación, floculación, aguas residuales, Cromo (VI).

\section{SUMMARY}

In order to generate the necessary tools to carry out the testing removal of chromium(VI) in wastewater from the galvanic industry, the data gathering is performed from a first prototype of equipment for this purpose. Once the data is obtained, the necessary changes are specified and it allows the improvement of the equipment and increases the potential for using it in the determination of working parameters on electrocoagulation.

The analyzed parameters were voltage source, current controller, temperature thermometer, and $\mathrm{pH}$ because they are the parameters to be standardized.

Throughout the textcan observe the changes made on the initial prototype whose main concept is handling electrocoagulation, to decrease the amount of metals in wastewater. An interesting outcome of analyzing among others, energy is therefore based on this consumption was calculated final value of energy versus the environmental cost, which certainly proves to be an important input for use in metal removal heavy and opens a possibility to continue with further studies of this type for the design of new reactors more efficient and helpful in the industry in general, which will help greatly in the reduction of environmental pollution.

Keywords: Electrocoagulation, flocculation, residual water, Chromium (VI).

\section{INTRODUCCIÓN}

Los residuos líquidos de origen industrial son una fuente de contaminación, si éstos líquidos no son tratados antes de su vertimiento pueden generar problemas de grandes dimensiones para nuestro entorno y ambiente. Debido a que pequeñas concentraciones generan un impacto ambiental alto y en razón a que las sustancias son solubles en mayor o menor medida, en un solvente polar como el agua, resulta difícil retirarlos de la misma.

La electrocoagulación, es uno de los métodos fisicoquímicos más empleados en la depuración de aguas residuales contaminadas con metales, te es un procedimiento simple y eficiente donde el agente de floculación se genera por electro oxidación de un ánodo de sacrificio, generalmente hecho de hierro 0 aluminio. En este proceso, el tratamiento se realiza sin añadir ningún agente químico 
coagulante o floculante, de tal manera que se reduce así la cantidad de los lodos que se deben eliminar [1]. La electrocoagulación es una técnica que ha sido utilizada con éxito para tratar desechos de aceite, con una eficiencia de remoción tan alta como el $99 \%$ [2], [3]. Un éxito similar se obtuvo en el tratamiento de las soluciones que contienen colorantes [4] agua potable [5], urbanos y aguas residuales de restaurante [6], [7] y el nitrato o el flúor que contiene aguas [8], [9]. Muchos de los trabajos realizados en las últimas décadas [10], [11] han demostrado que la electrocoagulación es una tecnología eficaz para el tratamiento de soluciones que contienen metales pesados.

Algunos aspectos de diseño a tener en cuenta, para implementar la técnica de electrocoagulación, están relacionados con el tamaño y geometría de la celda, los materiales y disposición de los electrodos, los cuales son fundamentales para lograr óptimos resultados con la aplicación del proceso [12]. Durante el 2010 y 2011, se ha trabajado en las instalaciones de la UMNG, en el mejoramiento del modelo de reactor de electrocoagulación propuesto en el 2009 [13], con el objetivo de mejorar su rendimiento disminuyendo consumo energético y facilitando las operaciones necesarias para remover cromo hexavalente de muestras de agua residual procedentes de una empresa del sector galvanotécnico de Bogotá.

Económicamente se considera que es viable la posibilidad de utilizar la electrocoagulación para retirar los contaminantes en el agua ya es más barata comparada con métodos antiguos en los cuales se debía emplear grandes cantidades de reactivos para llevar a cabo el proceso, lo cual implica que sea altamente costoso. Estudios reportados de trabajos en los cuales hacen remoción de $\operatorname{Cr}(\mathrm{VI})$, con electrodos de hierro, y remoción de $\mathrm{Cu}(\mathrm{II}), \mathrm{Ni}(\mathrm{II})$ empleando electrodos de aluminio, presentan gran efectividad y bajo costo en los procesos de electrocoagulación [14].

El electrodo de desgaste más favorable para la electrocoagulación es el hierro. Uno de los métodos tradicionales para remover cromo consiste tratar la muestra para reducirlo a $\mathrm{Cr}(\mathrm{III})$, con el fin de luego precipitarlo en medio básico; de esta manera se han obtenido una menor cantidad de lodos ricos en este metal producidos en el proceso químico [15]. El coagulante se forma por las reacciones de oxido reducción que ocurren entre los electrodos y el medio acuoso y son promovidas por la corriente eléctrica [15].

La celda electroquímica o reactor para la electrocoagulación puede ser tipo batch o continúo. En su forma más simple el reactor tipo batch es una cubeta en la cual, los electrodos se disponen en forma de placas paralelas conectadas en serie a una fuente de voltaje [15].

El volumen de la celda ocupada por los electrodos se define como zona de reacción, en esta, se dan los procesos de desestabilización de partículas, coagulación y sedimentación. Los lodos formados pueden depositarse en la parte superior de la celda cuando su densidad es baja, o precipitar cuando su densidad es alta, para luego ser removidos. El reactor tipo bach tiene importancia a nivel 
de laboratorio ya que permite el estudio del proceso y los parámetros a ser controlados.

\section{MATERIALES Y MÉTODOS}

Los análisis fueron realizados en el laboratorio de calidad de aguas de la Facultad de Ingeniería y en los laboratorios de Química, Facultad de Ciencias Básicas y Aplicadas de la Universidad Militar Nueva Granada, sede calle 100, en Bogotá D.C.

El prototipo se construyó en acrílico, con capacidad para $3.5 \mathrm{~L}$ de aguas residuales galvánicas en una única zona de operación, y con un conjunto de seis placas metálicas intercaladas, tres de hierro y tres de aluminio. Se conectaron 3 fuentes de poder (Erasmus EF-055) en paralelo, a tres juegos de placas, (cátodo de aluminio y ánodo de hierro), el valor de voltaje se alcanzó con un multímetro Uni-T (UT) 60A, los valores de corriente se obtienen con una pinza amperométrica Uni-T (UT) 201, la agitación magnética se llevó a cabo con una plancha de agitación Wisestir MSH2OD a 500 rpm, la conductividad y la temperatura se tomaron con un equipo EZDO PL700AL, el pH fue valorado con un $\mathrm{pH}$-metro Orion 3-Star Plus $\mathrm{pH}$ Benchtop Meter de Thermo Scientific, en cada una de las tres replicas se tomaron datos durante una hora de funcionamiento del reactor. Figura 1.
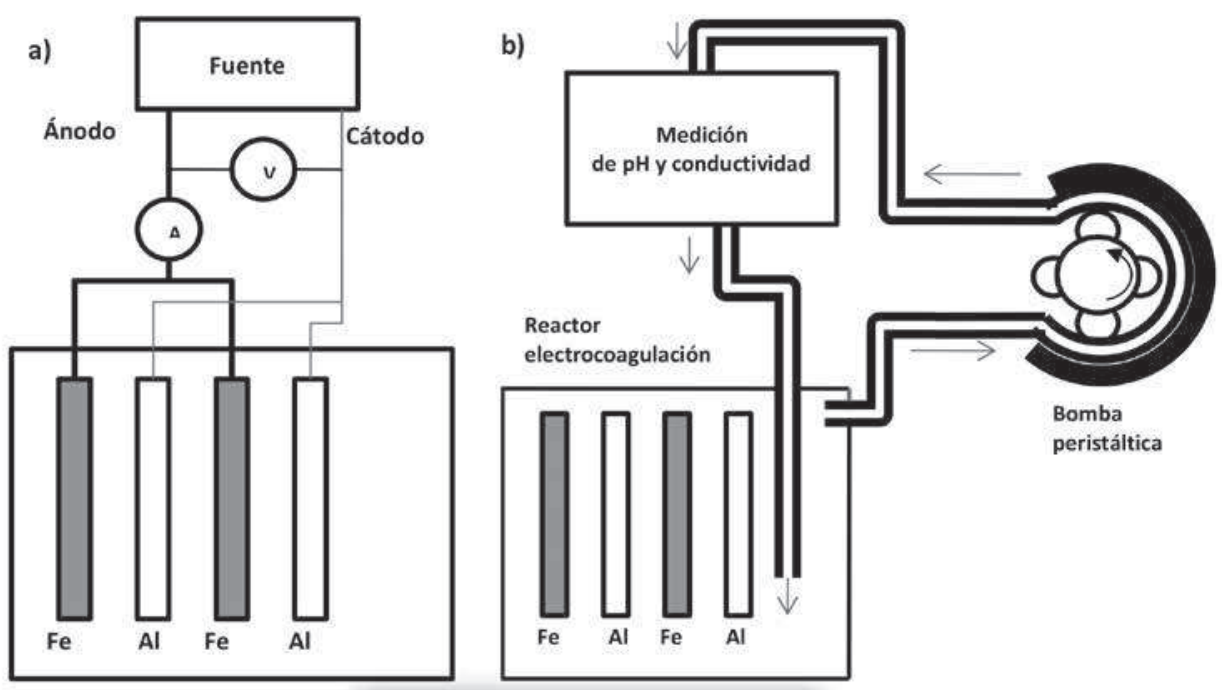

c)

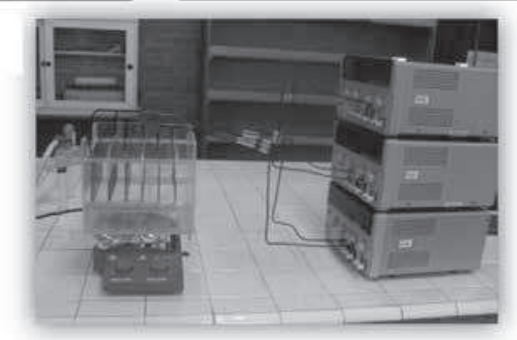

Figura 1. a) Plano eléctrico del reactor para electrocoagulación, b) Plano de flujo para solución en el reactor para electrocoagulación y c) Prototipo de celda de electrocoagulación. 
La solución acuosa entre las placas metálicas que actúan como cátodo (donde ocurre la reducción, en aluminio) y ánodo (donde ocurre la oxidación, en hierro) presenta determinada resistencia eléctrica la cual se verá alterada por la cantidad de iones disueltos, a los cuales se les hace seguimiento, teniendo en cuenta la conductividad eléctrica de la solución. Este valor se mide instrumentalmente, sometiendo la solución a un voltaje y determinando la cantidad de corriente que transita por el circuito. La medida nombrada puede alterarse por la existencia de un campo eléctrico exterior como el que generan las placas de hierro y aluminio.

La concentración de iones hidronio en la solución es determinante para la conductividad de la solución y dependiendo de esta concentración se ven favorecidas, disminuidas o a veces inalteradas las reacciones químicas, como en el caso de las oxidaciones y reducciones, la concentración de iones hidronio se mide indirectamente por la diferencia de potencial entre dos soluciones, por ende es una medida eléctrica y también está afectada por los campos eléctricos cuando estos se encuentran en el medio al cual se está midiendo el pH.

Al medir el voltaje y la corriente, mientras transcurre el tiempo se puede determinar la cantidad de vatios empleados, es decir, cuanta energía es consumida por el sistema. La energía absorbida por el sistema debe ser igual a la cantidad de energía empleada por el mismo. La energía en el sistema se divide en: la que se utiliza para la reacción de óxido reducción, la que se con- sume para el aumento de la temperatura de la solución, la que se disipa en forma calórica desde el sistema hacia los alrededores, y otras de menor importancia.

Si se evalúa el cambio de la temperatura del medio para cada momento se puede hallar la cantidad de energía utilizada en el calentamiento de la solución y al realizar un gráfico, el cambio en la pendiente se puede atribuir a la energía disipada por el sistema hacia los alrededores.

La conductividad puede indicar si es o no favorable la adición de electrolitos fuertes que disminuyan la resistencia eléctrica de la solución en la cual se encuentran los analitos de interés.

\section{RESULTADOS Y DISCUSIÓN}

A continuación se presentará la revisión de los datos adquiridos en el reactor con las condiciones iníciales de operación:

Las mediciones y estandarización de los parámetros a tener en cuenta para el buen funcionamiento del equipo, fueron generadas empleando muestras de agua de características normales, efectuando tres réplicas, de las cuales se presenta el promedio de resultados en la tabla 1. Estos cambios fueron realizados a partir de un prototipo inicial, en el cual se tomaron tres placas de hierro y tres placas de aluminio, con el fin de determinar la cantidad de material que se pierde en cada electrodo y con ello la cantidad de electrones que hacen parte de la oxidación del hierro, para de esta manera los costos del proceso. 
UNIVERSIDAD MILITAR NUEVA GRANADA - CIENCIA E INGENIERÍA NEOGRANADINA

Tabla 1. Pesos de placas obtenidos durante el tratamiento de remoción.

\begin{tabular}{|c|c|c|c|}
\hline $\begin{array}{c}N^{\circ} \text { de } \\
\text { Placa }\end{array}$ & $\begin{array}{c}\text { Peso Al } \\
\text { (antes ) g }\end{array}$ & $\begin{array}{c}\text { Peso Al } \\
\text { (después) g }\end{array}$ & Área cm2 Al \\
\hline 1 & 143,01 & 142,92 & 119,0 \\
\hline 2 & 142,39 & 142,47 & 110,5 \\
\hline 3 & 141,65 & 141,51 & 107,1 \\
\hline 1 & $\begin{array}{c}\text { Peso Fe } \\
\text { (antes ) g }\end{array}$ & $\begin{array}{c}\text { Peso Fe } \\
\text { (después) g }\end{array}$ & Área cm2 Al \\
\hline 2 & 387,89 & 387,77 & 119,0 \\
\hline 3 & 376,46 & 376,39 & 119,0 \\
\hline
\end{tabular}

El resultado esperado respecto al cambio en el peso de las placas de hierro, debía ser una disminución del mismo, a medida que el proceso evoluciona. Pero de acuerdo con los datos obtenidos se observó que contrario a lo esperado, los pesos se incrementaron en los mismos, lo que puede deberse a que inicialmente estaban desnudos los electrodos y posteriormente quedaron cubiertos con una capa rojiza de óxido férrico.

Como la corriente permanece prácticamente constante al igual que el voltaje se puede determinar la energía suministrada por la fuente:

$$
36,1 V * 12.48 A=451 W
$$

Dada la potencia anterior, en una hora es $450 \mathrm{~W} / \mathrm{h}$ y este valor obtenido es preciso para determinar el costo del proceso, lo anterior teniendo en cuenta la cantidad de energía eléctrica consumida y el lugar en donde ha sido utilizado el proceso de electrocoagulación, ya que en Bogotá, el valor de kW de energía se encuentra estratificado.
Por otra parte en nuestro reactor se colocaron tres juegos de placas, entendiendo por juego una placa de hierro y una de aluminio, los electrodos se conectaron en paralelo como se muestra en la figura 2 , que esquematiza la forma de conexión de los electrodos.

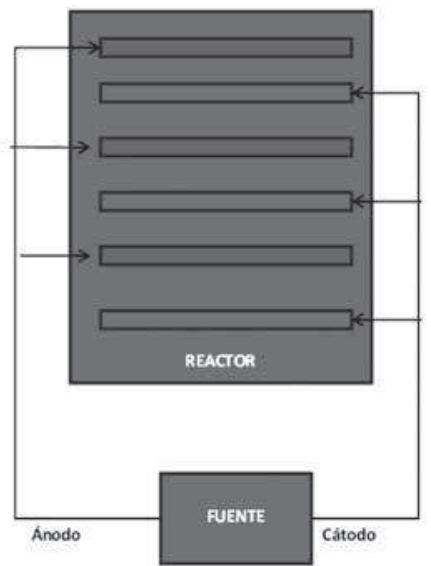

Figura 2. Conexión de los electrodos en el reactor.

A continuación se presentan los gráficos realizados con los respectivos datos para el montaje en las condiciones iniciales:

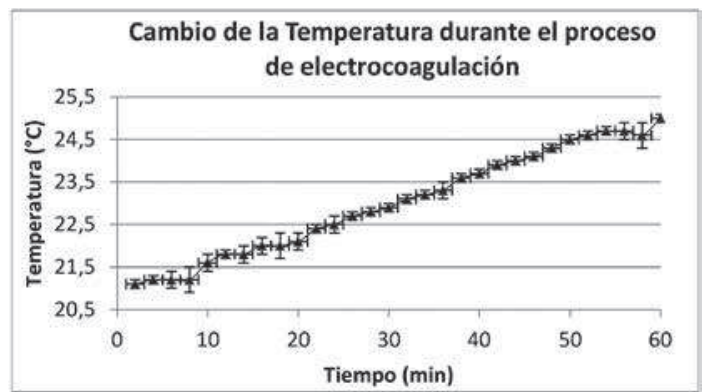

Figura 3. Comportamiento de la temperatura con respecto al tiempo.

El comportamiento que se observa en la gráfica 3, para el seguimiento de la temperatura, corresponde con lo esperado, ya que una parte de la energía que es empleada en el sistema se transforma en energía térmi- 
ca acumulada en el mismo, se espera que a medida que aumenta la temperatura se reduzca la pendiente con tendencia a estabilizarse. La reducción en la pendiente es la esperada debido a que se presenta una mayor diferencia entre la temperatura del reactor y el ambiente.

Para calcular la energía absorbida por el agua se parte de la diferencia de temperaturas $\left(t_{f}-t_{i}\right)$ y la capacidad calorífica del agua, por ser el principal componente:

$$
\Delta T=4,1^{\circ} \mathrm{C}
$$

Al realizar el cálculo para el recipiente de capacidad 3.5 litros, completamente lleno, se encontró que se requieren en total 32,8 $\mathrm{kCal}$, lo cual equivale a $1,66 \times 10-2 \mathrm{kWh}$.

Este resultado es un valor relativamente bajo, comparado con los consumos de energía registrados, por tanto es un indicativo de la poca la energía disipada en forma de calor.

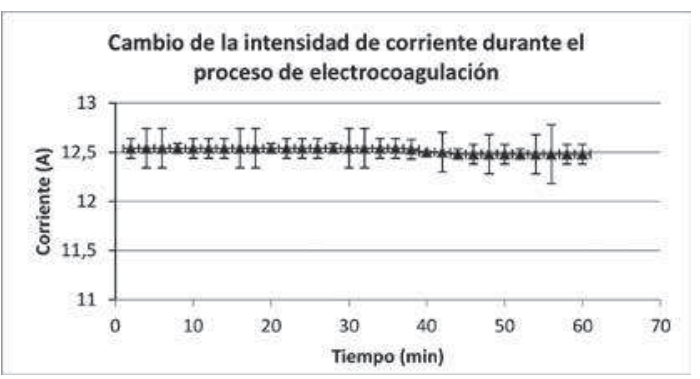

Figura 4. Variación de la corriente con respecto al tiempo.

La corriente no presenta ningún cambio significativo, el salto que se observa en la gráfica de la figura 4 es prácticamente insignificante, según se puede determinar de acuerdo con los valores presentados en la escala que se encuentra en el eje vertical izquierdo.

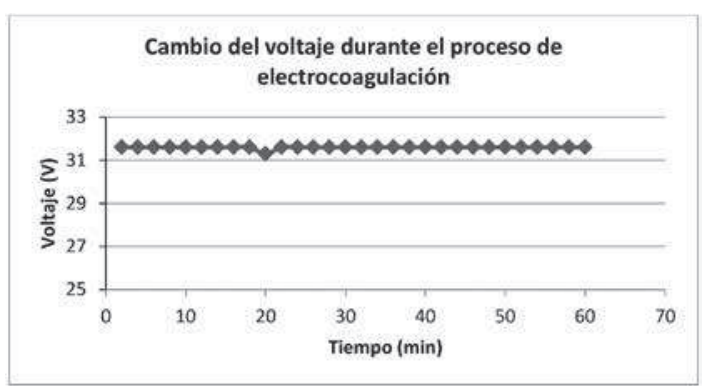

Figura 5. Variación del voltaje con respecto al tiempo.

De acuerdo al resultado observado en la gráfica de la figura 5, no se presenta cambio en los valores de voltaje, indicando que este permanece constante durante todo el proceso. Se omite la desviación estándar debido a su bajo valor.

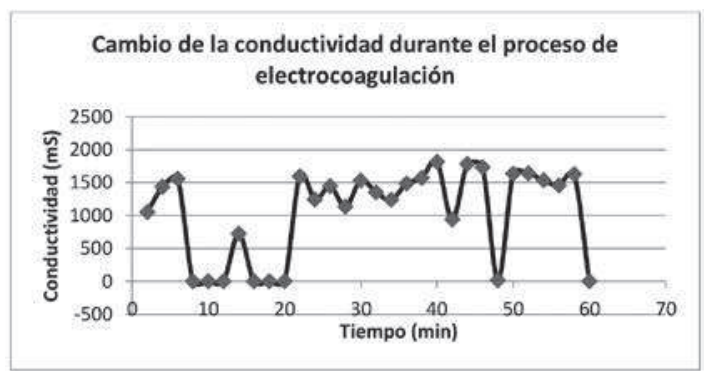

Figura 6. Relación de conductividad con respecto al tiempo.

La gráfica de la figura 6 presenta la relación de conductividad, respecto al tiempo, esta muestra un comportamiento irregular, y en algunos datos se observa variación en tres órdenes de magnitud, razón por la cual se sugiere disponer una toma de muestras continua, con el fin de evitar que el campo eléctrico generado en el reactor interfiera con el campo eléctrico generado por el instrumento de medida para la conductividad. 
Los cambios son muy bruscos por tanto no se presenta la desviación estándar ya que es muy alta.

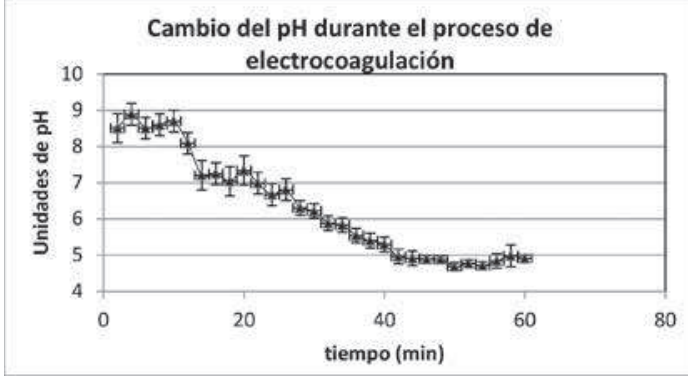

Figura 7. Relación del pH con respecto al tiempo.

El pH presenta reducción a medida que avanza el proceso (ver gráfica 7). La deviación estándar es baja y permite afirmar que los cambios en el pH son representativos.

Después de observar el comportamiento arrojado por las gráficas para los parámetros analizados en función del tiempo se recomienda para el funcionamiento del equipo:

- $\quad$ Evitar que la electricidad utilizada para la electrocoagulación interfiera con los instrumentos que se utilizan para la toma de datos.

- Para poder cumplir con lo expuesto en el párrafo anterior se sugiere colocar en recipientes diferentes la electrocoagulación y los instrumentos de medida.

- $\quad$ El muestreo debe realizarse en forma continua y evitando el contacto de la solución con sustancias que alteren la composición de la solución a tratar.

- Con el fin de cumplir lo anterior se sugiere el uso de una bomba peristáltica de baja velocidad y mangueras siliconadas.

- Para reducir la complejidad de la bomba se estima conveniente colocar el recipiente de medición a una altura mayor que la del reactor lo cual permite el uso de una manguera de ascenso para la solución y el líquido regresa al reactor por gravedad (caída).

Se diseña el cabezal de la bomba peristáltica que permite su ensamble. (Figura 8).

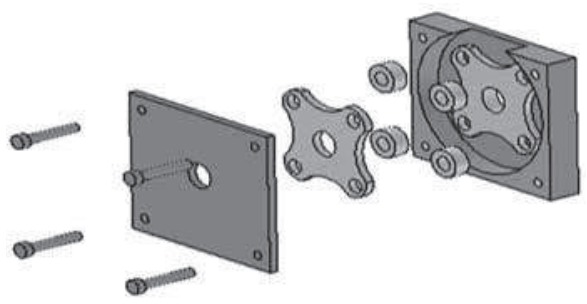

Figura 8. Plano de la bomba peristáltica.

La fabricación de una bomba peristáltica permite el mejoramiento del equipo de electrocoagulación. Se indica la forma de montaje de las fuentes eléctricas con el fin de minimizar la posibilidad de sobrecargas, cortos circuitos e interferencia entre las mismas. Lo anterior orienta el mejoramiento del equipo para electrocoagulación y la disposición del mismo al igual que permite la toma de decisiones para la requisición de datos y posibles adquisiciones a futuro. En las fotografías (Figuras 9 y 10) se muestran los cambios realizados sobre el prototipo para la recolección de metales en aguas residuales, se adiciona una fuente de voltaje, controlador de corriente, medidores de temperatura y $\mathrm{pH}$ debido a que son los parámetros a estandarizar. 


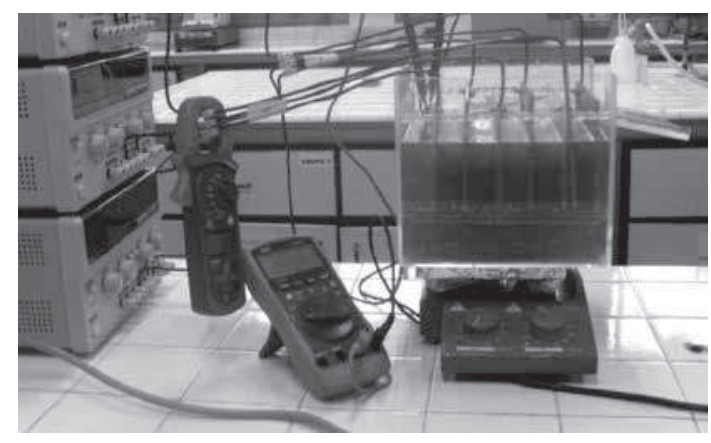

Figura 9. Reactor (medición de corriente).

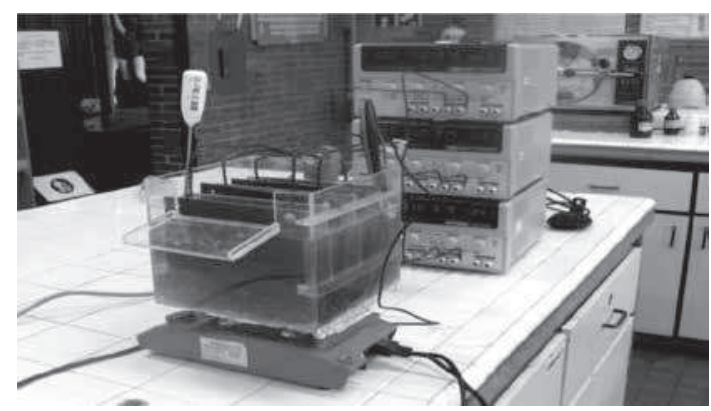

Figura 10. Reactor (medición de temperatura y pH)

\section{CONCLUSIONES}

Una vez fueron analizadas las variables mencionadas durante el proceso, se definen para este reactor un voltaje y una corriente constantes en razón a que la potencia indica un consumo de energía aceptable para el empleo de este equipo.

Para la estandarización de la conductividad eléctrica se debe realizar la toma de medida en un recipiente aislado eléctricamente del reactor.

El pH disminuye a medida que pasa el tiempo por tanto es necesaria la adición de sustancias amortiguadoras de $\mathrm{pH}$.
Una rebaja en el gasto energético para este proceso, puede contribuir a la disminución en el costo general del proceso y por ende su posible implementación en la industria, con el fín de utilizarse como una alternativa viable en la remoción de metales provenientes de aguas residuales.

El presente trabajo indica que este primer acercamiento en la elaboración de un prototipo (Reactor) cuya finalidad es la remoción de metales en aguas residuales, se puede mejorar y transformar a medida que sean fortalecidos y profundizados estudios que estén enfocados en controlar y determinar todas las variables implícitas en el proceso de electrocoagulación, por su parte, como se nota en las diferentes gráficas cada vez que se relacionen las múltiples variables con respecto a la eficiencia del proceso y se logre un cambio a partir de estudios previos sin duda se abrirá la posibilidad para su aplicación en el tratamiento de aguas con el fín de reducir los niveles de contaminación y así contribuir a la conservación del medio ambiente.

\section{BIBLIOGRAFÍA}

[1] Cenkin V and Belevstev A, (1985), Electrochemical treatment of industrial wastewater. En: Eff. Water Treat., vol. 25, no. 7, pp. 243-249, 1985.

[2] Biswas N and Lazarescu G, (1991), Removal of oil from emulsions using electrocoagulation. En: Journal environmental, vol. 38, pp. 65-72.

[3] Renk R, (1988), Electrocoagulation of tar sand and oil shale wastewater. En: Energy Prog, vol. 8, pp. 205-208. 
[4] Do J and Chen M, (1994), Decolourization of dye-containing solutions by electrocoagulation. En: journal of electrochemical, vol. 24, pp. 785-790.

[5] Vik E, Carlson D, Eikum A, and Gjessing E, (1984), Electrocoagulation of potable water. En: Journal water research, vol. 18, pp. 13551360.

[6] Pouet M and Grasmick A, (1995), Urban wastewater treatment by electrocoagulation and flotation. En: Water Science and Technology, vol. 31, pp. 275-285.

[7] Chen X, Chen G, and Yue P, (2000), Separation of pollutants from restaurant wastewater by electrocoagulation. En: Separation and Puriffcation Technology, vol. 19, pp. 65-76.

[8] Koparal A and Ogutveren U, (2002), Removal of nitrate from water by electroreduction and electrocoagulation. En: Journal of Hazardous Materials, vol. 89, pp. 83-94.

[9] Shen F, Chen X, Gao P, and Chen G, (2003), Electrochemical removal of fluoride ions from industrial wastewaters. En: Journal of Chemical \& Engineering, vol. 58, pp. 987-993.

[10] Mrozowski J and Zielinski J, (1983), Studies of zinc and lead removal from industrial wastes by electrocoagulation. En: Environment Protection Engineering, vol. 9, pp. 77-85.

[11] Kumar P, Chaudhari S, Khilar S, and Mahaja S, (2004), Removal of arsenic from water by electrocoagulation. En: Chemosphere, vol. 55, no. 9, pp. 1245-1252.

[12] Arroyo M.G, Pérez $V$, Herranz $M$, and Montañés $J$, (2009), Effect of pH and chloride concentration on the removal of hexavalent chromium in a batch electrocoagulation reactor. En: Journal of Hazardous Materials, vol. 169, pp. 1127-1133.

[13] Morales P. B and Acosta N. G., (2010), Sistema de electrocoagulación como tratamiento de aguas residuales galvánicas. En: Ciencia e Ingeniería Neogranadina, vol. 20, no. 1, pp. 3344.

[14] Heidmann I and Wolfgang C, (2008), Removal of $\mathrm{Zn}(\mathrm{II}), \mathrm{Cu}(\mathrm{II}), \mathrm{Ni}(\mathrm{II}), \mathrm{Ag}(\mathrm{I})$ and $\mathrm{Cr}(\mathrm{VI})$ present in aqueous solutions by aluminum electrocoagulation. En: Journal of Hazardous Materials, vol. 152, pp. 934-941.

[15] Mollah M, Schennach R, Parga J, and Cocke D, (2001), Electrocoagulation (EC) - Science and applications. En: Journal of hazardous materials, vol. 84, no. 1, pp. 29-41. 